\title{
Technical Challenges for Integrated Design and Delivery Solutions
}

\author{
R.W. Amor \\ University of Auckland, Auckland, New Zealand
}

\begin{abstract}
Conceptually, the development of an extensible infrastructure for Integrated Design and Delivery Solutions (IDDS) would appear to be a straight-forward matter. However, there are few current implementations in existence, and the majority of those that do exist are bespoke developments which support a restricted number of processes and analyses. This paper characterizes the wide range of technical challenges which are faced by those delivering on the promise of integrated design and delivery solutions. It examines the levels of IT support that can be offered for aspects such as: collaborative work processes; repositories of integrated data; management of information integration; and knowledge management processes. Alongside each of these challenges it identifies current approaches to supporting IDDS, both at a commercial level with tools that can be deployed today, and from the viewpoint of researchers working on future improvements to the IDDS ideal. The last part of this paper establishes a technology foresight for the technical development of IDDS. Providing a view of the technical future of IDDS over the coming decades with projections of the pathways that will lead to adoption of various forms of IDDS and the major obstacles where the dearth of solutions will delay the uptake of IDDS.
\end{abstract}

\section{INTRODUCTION}

In developing their IDDS theme, CIB have chosen the following definition of IDDS:

"Integrated Design and Delivery Solutions use collaborative work processes and enhanced skills, and integrated data, information, and knowledge management to minimize structural and process inefficiencies and to enhance the value delivered during design, build, and operation, and across projects." (CIB 2009)

While recognizing that an IDDS comprises three major aspects: people; process; and technology, the people and process aspects of an IDDS will not be considered in this paper. Instead the focus is fully on technical aspects of an IDDS.

Limited forms of integration are offered through BIM-based approaches offered by major CAD vendors. These approaches are typically vendor specific and tie together a small number of design tools (in comparison to the thousands available in the marketplace), and are unlikely to be the set required by any particular grouping of professionals involved in a construction project. Creating a wider integration platform usually requires uniquely qualified individuals to be available in one of the companies involved in the project. Some demonstration of interoperability utilizes the IFC data model standard, which cuts across CAD vendors, though still only providing support for a limited set of construction processes, and still requiring uniquely qualified individuals in each company to ensure the integrity of data exchanged. Due to the limited number of applications supported in BIM, or interoperable solutions, the practice of manual re-entry, and checking, of data between applications is common. Where automated information exchange is practiced the common experience is of loss of information and no guarantee of the semantic integrity of models being exchanged. The usual characterization of information management with current BIM and interoperability solutions is to a document management system, where a complete model is exchanged and the only level of information management is achieved through interrogation of the different versions of the complete model being passed between project participants.

Interoperability will be seen to be ubiquitous in the industry when practitioners do not understand that there is a complex and sophisticated technology underlying their software tools. When there will be a seamless connection between any two software tools gathering, and updating, the particular view of information required for any particular process in the project. Then specialist coders will not be required by companies to undertake project-based information exchange, though there will be roles for an inte- 
roperability manager within companies to establish the right approach for the software tools required for a project.

Reaching this future state requires further significant work on many aspects of information exchange already developed over the last two decades. The data models which underlie all information exchange require significant development to cover all major processes as well as an enormous effort in defining the information views required by particular classes of application and standard processes. Sophisticated approaches to model and view-based information management need to be developed which cope with project structures and even the processing times common in the industry. Software developers will need to put significant resource into ensuring the adequacy of their products to exchange semantically consistent views of buildings under most conditions. The software tools developed here will also need to tie into the systems which support knowledge management within companies and for the industry.

This paper is based on a keynote presentation given at the $1^{\text {st }}$ International Conference on Improving Construction and Use Through Integrated Design Solutions (Amor 2009).

\section{IDDS TECHNICAL TIERS}

To focus on the necessary technical needs of an IDDS we consider the range of technologies in a layered fashion as shown in Figure 1.

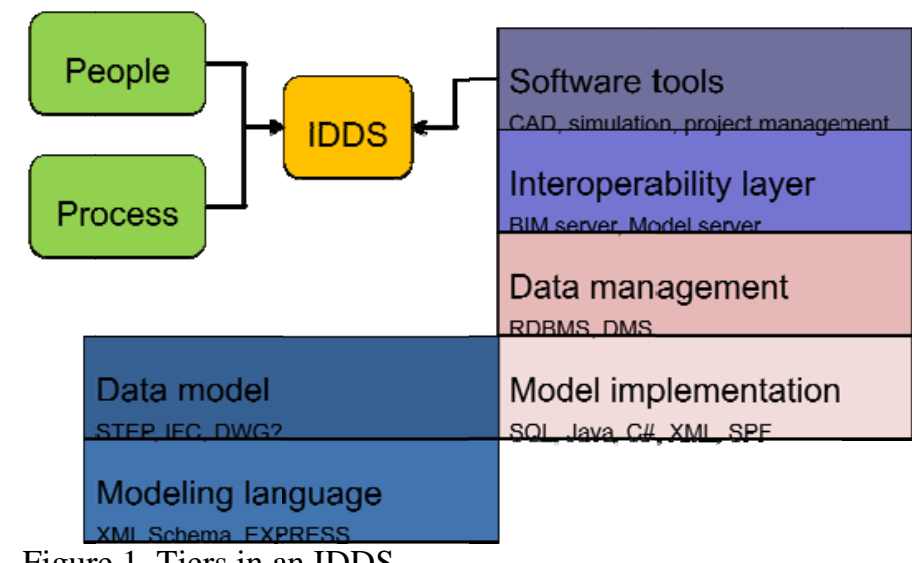

Figure 1. Tiers in an IDDS.

At the lowest level in this model we identify abstract aspects of modeling as specified in one of many popular modeling languages. Of particular interest to the $\mathrm{A} / \mathrm{E} / \mathrm{C}-\mathrm{FM}$ industries is the EXPRESS data modeling language and the more modern and less specialized XML Schema language.

Through the use of such modeling languages domain experts are able to develop and agree upon domain specific data models. This is the work undertaken by groups such as ISO and IAI for A/E/C-FM domains.
Once data models are specified and agreed implementers make choices about how the data model is implemented through deployment in a particular programming language or data description language. These languages have different strengths and weaknesses and hence different choices suit particular implementations to a greater or lesser degree.

Data management choices determine where data resides in an IDDS and again provide for differing levels of support for particular operations on the data for a project.

The interoperability layer provides the range of services that enable software tools to share data across the IDDS and ensure the semantic integrity of the data as it moves from user to user and process to process.

Finally, software tools provide the particular functionality that enables users to action a particular process in the design and delivery of a project.

\section{DATA MODELS}

\subsection{Data Model Progress}

Over the last few decades there has been significant progress in the development of open data models for the A/E/C-FM industries (Eastman 1999). Within the ISO STEP developments the standard 10303:255 (Building elements using explicit shape representation) has been published, though it currently seems to have little commercial take-up. The most commercially successful standard development initiative has been that of the IAI (2009) with their IFC standard (now reaching version $2 \mathrm{x} 4$ ). As well as being developed as an industry standard it has been submitted as a PAS within ISO. The IFC $2 \times 3$ has seen fairly strong take-up as an import/export option amongst the major CAD vendors and more limited take-up in other software tools for the industry.

There are also a range of closed data models which represent the developments made by individual software developers for their particular tools. For instance, the BIM (Building Information Modeling) approach which is currently popular in the industry requires a sophisticated data model within each BIM software tool. This leads to separate data model developments for CAD systems such as Revit, ArchiCAD, ADT, Microstation, etc. The data models each company develops is only utilized by tools which are tightly bound to the particular CAD system, though the DWG format from Autodesk may be seen as a quasi-standard with its reverse engineering through the Open Design Alliance (2009).

The IFC data model represents over a decade of international development effort with a focus on providing support for major processes required in the $\mathrm{A} / \mathrm{E} / \mathrm{C}-\mathrm{FM}$ industries. The model now contains over 600 class definitions for a wide range of core 
building elements as well as non-tangible aspects of a construction project. This data model is still being developed to incorporate new processes and to provide links to associated domains such as GIS. Figure 2 provides the IAI's analysis of completeness of the standard in a range of areas as of IFC $2 \times 2$. Their self-assessment shows an average of around $80 \%$ completeness for many of the major disciplines and areas supported by the data model.

Figure 2. IFC data model progress (IAI 2009)

\subsection{Data Model Challenges}

While Figure 2 indicates $80 \%$ coverage for the processes and areas developed to date it is clear that there will be numerous challenges to the further development, and perhaps to notions of completion, of the data model. From a software engineering viewpoint (Sommerville 2006), it is expected that as the size and complexity of the data model increases the effort required to make changes and additions will increase substantially. This comes from many impacting forces. The effort required to become au-fait with the data model will steadily increase, requiring greater effort from experts in new disciplines to get to the point where they can contribute to the model. Checking the correctness of the data model after changes will also become more complex as the flow on effect of changes impacts a larger and larger number of processes and design tools which have implemented the standard. International validation of the new additions will become more difficult for both of the reasons highlighted above. Implementation of the updated data model will become more time-consuming (already estimated by Autodesk to require one person year per version), and a greater barrier to companies who have not yet started this process.

When new processes are considered against the existing IFC data model, for example fire engineering see Dimyadi et al (2008), the domain experts for that process take on the burden of understanding the data model to the extent that they can identify preexisting and missing structures. Dimyadi et al (2008) identified that the majority of the needs of fire engi- neering are supported by the existing IFC data model. However, several objects and attributes were required to be added to support the specific needs of the domain (e.g., notions of a fire source) and this required modifications of the data model, which would then need to be tested both by design tools in the fire engineering domain, as well as for their impact on existing design tools.

It is clear that there are many existing processes whose needs could be incorporated into the IFC data model, and that considerable time will be required to accommodate their needs in this model. There are also new disciplines emerging (e.g., communications engineer), and the data model will always have to evolve to accommodate the needs of these disciplines. One might posit the $80: 20$ rule to project what will be required to get to a complete data model. If we take 10 years to achieve $80 \%$ of the data model, then we could be looking at a further 40 years effort to get to a data model that covers the majority of processes we would wish to support.

When looking at what objects are specified within the data model there is not a distinguishing line between what might be added as an explicit object, and what might be left as classifications within an object type. One might question whether 600 classes is sufficient for a domain of the size and complexity of $\mathrm{A} / \mathrm{E} / \mathrm{C}-\mathrm{FM}$. Certainly, it is nowhere near the level of distinction and granularity we see in large classification systems, where somewhere around 15,000 distinct products are specified. However, it is unlikely that anyone in this domain will be arguing for a data model which does contain 15,000 or more class definitions. The question is what is the number that would be sufficient?

Few researchers are looking to develop a data model which is monolithic and covers all of the possible domains of interest to A/E/C-FM (Amor and Faraj 2001). There exist large and proven data models for other domains such as infrastructure, GIS, plant, furniture, etc which could be utilized when dealing with information needs which lead into those areas. Certainly when one looks at standards which have been successfully taken up in recent years in other domains these tend to be smaller and more agile standards (e.g., HTML, XML, etc). This would argue that research work dealing with ontologies and classifications (e.g., Christiansson et al 2008, Yurchyshyna et al 2007 and Grilo et al 2007) should have further consideration in competition to the work developing the single data model.

The notion of developing MVD (Model View Definitions) which specify the actual data needs of a particular domain or process as a subset of the complete IFC data model is an important step forward for a large data model. This is very similar to the approach of Application Protocols (AP) in the ISO STEP community. To date a single MVD has been specified by IAI, though many more are likely to be 
added as design tools mature and the size of data files drives the need to pass just relevant data for particular processes. Identifying the subset of the complete IFC data model required for a MVD is a major task in its own right, requiring domain experts with significant knowledge of the IFC data model. Adding MVD specifications will add to the complexity of the development of the IFC data model. As new structures and objects get added to the base IFC data model all existing MVD will need to be checked against the new model to ascertain whether they need to be modified for the new structures.

\section{DATA MANAGEMENT}

\subsection{Data Management Progress}

The management of data for individual design tools and CAD packages is an area which receives considerable attention from developers of those packages. Representations and storage structures are chosen to ease the process of accessing required data, and to minimize the amount of data to be transferred between applications. This approach also works well where several applications utilize the same optimised representation of building information (e.g., a DWG file).

\subsection{Data Management Challenges}

The use of standard representations for the transfer of information between systems creates a large data management challenge. Unlike proprietary formats which are optimized for use by a particular application (e.g., with indices to particular types of objects) the standard formats currently utilized for ISO and IAI standards (e.g., STEP Physical File (SPF) and $\mathrm{XML}$ ) must be fully populated with the complete building's data and completely processed by an application to extract the required information. There are two issues with this approach.

One is the size of the files created through this process. An ASCII version of a database of information, especially in a verbose format such as XML, greatly increases the size of file required to encode building information. The consequence being files of great size even for a modest construction project. Transferring these files between the various project participants then requires specialist support (e.g., not amenable to most email transfer protocols). As the IFC data model increases the number of categories of building related data which can be described the amount of data transferred increases. As an increasing number of applications are able to deliver information in the standardised format then the amount of data collated for the project increases. With data files easily reaching hundreds of megabytes the time and bandwidth required to transfer a building's description becomes a bottleneck for collaboration.
Secondly, is the time required to process very large data files. Data files with hundreds of thousands of objects must be parsed and reassembled into the data structures utilized by each design tool and CAD system requiring the data. This is a significant processing task, prone to error (see section 5.2), and again becomes a bottleneck in the collaboration common on projects.

In current approaches to data management the whole building model is transferred in the file. Once MVDs for major domains are created this will greatly reduce the amount of the building model which is every transferred. However, with any approach there is a problem in ensuring that all applications preserve the data which is transferred through to them for subsequent transfers. Currently, for most design tools the requirement is that they store and manipulate significant chunks of data which are of no interest (e.g., structural design tools do not require information outside of their analysis domain). This leads to design tools dropping data and subsequent data transfers losing information from the original files. To cope with this actuality currently requires vigilance on behalf of project managers and a significant manual reentry of data.

Once MVDs are in place the requirement for information transfer and processing will be significantly reduced. However, this comes at the cost of handling the subsequent mergers of overlapping and modified construction information. While transactional approaches are available which can guarantee the consistency of overlapping data model updates in these circumstances (Gray and Teuter 1993) they are tedious in practice. In particular the impact of these techniques is to move processes away from concurrent execution towards serialized execution and often require significant user input to resolve clashes in data. Support for such user involvement in data conflict resolution is not a feature found in the many applications which utilize such data.

There are a wide range of standard data management issues which are not being well supported by current approaches. These include agreements on the ownership of data created during a project and especially protection of the intellectual property rights of those involved in a project. Identifying the provenance of the data in a building model is poorly supported and will require the complete creation data and change history to support the range of legal processes associated with construction processes. Support for automated change propagation is not available in design tools and CAD systems, and even automated notification of change is absent in most tools. Approaches to archiving of the complete data model are also not well supported, except as a file to be managed as any other file is in a backup policy for a computer or site. 
Current uses of data models do not utilize very much of the site information which is potentially available, and consequently do not support their inclusion well. Real-time monitoring of a site introduces a significant flow of data into a data model. This could include video feeds of the site, sensor data which provides continuous readings, a more frequent use of RFID data for tracking and placement of objects on site, site photographs, etc. Any one of these data feeds having the potential to generate volumes of data which will swamp the data currently found in construction data files.

\section{INTEROPERABILITY}

\subsection{Interoperability Progress}

BIM providers, and the IAI, promote their ability to tie a small group of applications together in a controlled and tightly coupled manner (IAI 2009). The suite of applications interoperating are usually chosen to provide coverage of major processes within $\mathrm{A} / \mathrm{E} / \mathrm{C}-\mathrm{FM}$ and hence, if the specified design tools are purchased, coverage of the supported processes is possible (Eastman et al 2008). Design tools and CAD systems interoperating in this manner are usually tested and certified to be working at a certain guaranteed level of compatibility, providing reassurance on a project that process interoperability will be efficient. There can be difficulties with this approach when project teams come together and find that they are not utilizing the tools required for such interoperability, and there is usually significant resistance to changing away from tools that are well known within an organisation.

\subsection{Interoperability Challenges}

The current state of play of BIM and IFC-based interoperability provides a view of what can be achieved with significant effort on behalf of the industry. However, what has been observed as perhaps the major barrier to increasing the level of interoperability for $\mathrm{A} / \mathrm{E} / \mathrm{C}-\mathrm{FM}$ is the great difficulty in providing correct mappings of the data models between CAD systems and other design tools. It is clear that problems as highlighted in Figure 3 are common with the current state of play of interoperability. The impact of inadequate interoperability in an industry has been identified as a major barrier to many innovations (NIST 2004).

Correctly specifying the mapping between a CAD system's or design tool's representation of a building and that of the transfer format (e.g., IFC) is obviously a major issue. As with correctly specifying the requirements of a software system (Sommerville 2006) it is recognised that a completely correct specification is not possible to achieve. Even for well documented data model specifications such as IFC there are many implicit assumptions about the classes being specified which are open to interpretation. This will also be true for the data model of the application being mapped to. On top of these incomplete understandings of the data models it will not be possible to correctly specify all the required mappings for any given specification. A problem which is analogous to correctly coding software to a known specification.

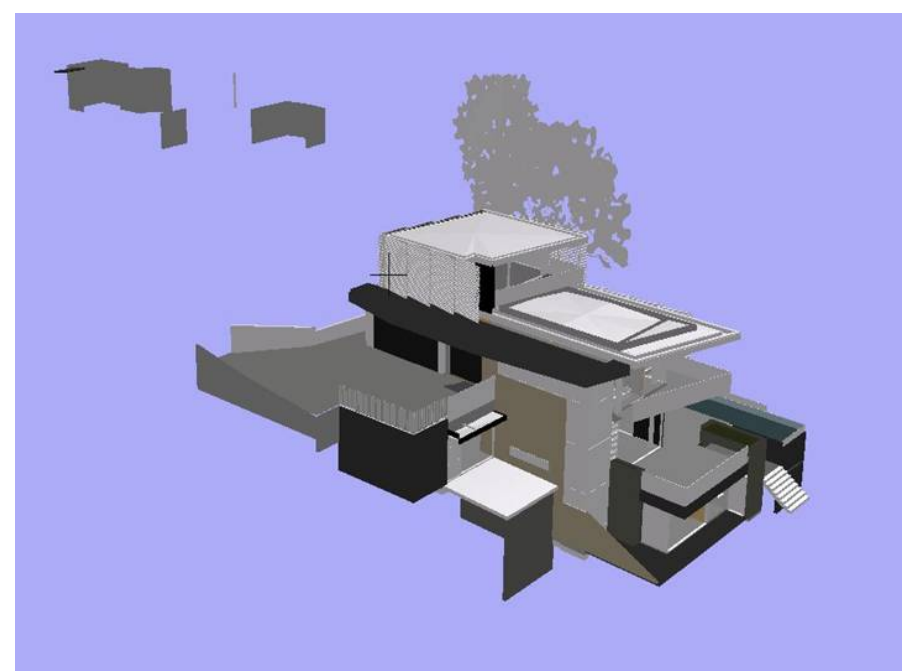

Figure 3. Poor interoperability due to incorrect mappings.

Several research projects have looked at the existing levels of interoperability for CAD systems and design tools and report significant errors in the mapping implementations, leading to issues as highlighted in Figure 3 (Amor and Ma 2006, Amor et al 2007, Lipman 2006, Ma et al 2006, Pazlar and Turk 2006). To retain the confidence of users of interoperable systems there needs to be further focus on addressing such issues. One important step towards managing such issues is the level of conformance testing which is required for a system to be deemed interoperable. Developing adequate testing approaches is a well recognised problem in the software industry as a whole (NIST 2002). Current approaches tend to favour the software developers who must balance their development efforts against significant conformance testing efforts. However, IAI (2009) have recognised that new approaches to conformance testing need to be introduced and are exploring more stringent testing to receive certification.

A further sign of the lack of confidence in interoperability is the existence of model checkers as stand-alone applications for purchase and use on construction projects. Tools such as Solibri (2009) provide an invaluable service in identifying problems during data transfers and finding solutions to those problems. However, until the need for such applications disappears it is clear that interoperability problems have not been solved.

When envisioning interoperable solutions for $\mathrm{A} / \mathrm{E} / \mathrm{C}-\mathrm{FM}$ projects the major system architecture 
which is proposed is a star configuration. In this model there is a single central repository (or model server) of the complete building model and all other applications interoperate through this central repository. There are many benefits to such an approach which simplifies many aspects of interoperability, but it is unlikely to be the only architecture which is chosen. A federated architecture, which is common in complex multi-organisational database systems, is likely to be a common approach as interoperable tools become more sophisticated. Such an approach allows for data to be held within any organisation (resolving some ownership issues) and queries against the model have to draw data from a number of data stores (or model servers). Other architectures, such as a hierarchical architecture, may suit particular types of organisational groupings and will have to be accommodated in the approaches supported by interoperable tools.

A further issue which impacts on interoperability is that of knowledge management for an organisation, a project, and even the industry. While approaches to knowledge management are evolving (Kazi and Wolf 2006) they have not reached a particularly sophisticated level. Management of knowledge at all the levels required, such as individually, for particular processes, for an individual project, for a company, and at an industry or country level are not possible with current approaches. Many of the applications supporting knowledge management are adaptations of electronic current document management systems and provide little further functionality over the underlying system.

\section{SOFTWARE TOOLS}

\subsection{Software Tool Progress}

The A/E/C-FM industries are well served by software applications addressing various national markets and the wide range of processes in the industry. A project to identify A/E/C-FM software in 2000 identified over 4,500 individual software tools for the industry (Turk and Cerovšek 2000). With the advent of BIM new levels of functionality have reached the mass market (though many of these functionalities existed in much earlier tools, see Howard 1998). Collision detection is now a standard process in design stages of projects. The use of 4D tools either within CAD systems, or as standalone tools, is providing for better planning of on-site processes. 5D (cost) simulation is also becoming available in BIM-based products, adding another view onto the construction process. Parametric CAD is available with innovative tools to allow designs to be generated from sophisticated descriptive systems (Bentley 2009).

\subsection{Software Tool Challenges}

As well as being a testament to the strength of the $\mathrm{A} / \mathrm{E} / \mathrm{C}-\mathrm{FM}$ industries, the fact that there are over 4,500 software tools available is also a major barrier to IDDS. Autodesk have estimated that the implementation of an interface to a data model standard such as IFC requires one person year's effort. Multiply that across 4,500 software tools, and then across a new version of the data model being released every couple of years, the impact on the industry of supporting interoperability can be seen to be very significant. Supporting the development of interoperability without such an overhead for software developers would seem to be a necessary step forward, while ensuring that the quality of the implementation is to the level which enables surety in the industry.

Using a design tool or CAD system requires the user to make decisions about the design. The intent of the designer when making these decisions, and using these tools (e.g., detailing a generative component), is not readily captured in current systems. Until knowledge management tools support their users in capturing such information there will be a gap in the completeness of the building model which is handed across at the end of a project.

There is still a considerable gap between the results served by the wide range of simulation tools and the actuality of the completed structure. While it is clear that the sophistication of a building is of a level that can not be completely simulated, groups such as CIFE at Stanford University are embarking on research to close the gap between simulations of the modeled building and the observed performance of the completed structure.

Usability and human-computer interaction (Preece et al 2002) has not been a major research concern in A/E/C-FM over the last decades, though there are signs that this is changing with the impact of greater numbers of mobile devices on the construction site. Good usability has the potential to significantly improve the productivity of A/E/C-FM professionals in their daily tasks with software tools and mobile devices. Good usability also has the potential to reduce errors in the processes supported by software and hardware devices.

\section{CONCLUSIONS}

In choosing to move towards IDDS as an approach for the $\mathrm{A} / \mathrm{E} / \mathrm{C}-\mathrm{FM}$ industries there are a range of issues which impact on the people in the industry, the processes they undertake over a project, and the technologies that support them. When considering the range of technologies required for IDDS there is obvious support for many of the core areas identified. However, it is also clear that the technologies 
in place today are the first incarnation of what will be required in the future. For each layer in the technology stack required for IDDS there are significant challenges to be addressed before we can claim to have reached the level of support required by the people and processes in this industry.

\section{REFERENCES}

Amor, R. 2009. Technical Challenges for IDS, Keynote presentation, $1^{\text {st }}$ International Conference on Improving Construction and Use Through Integrated Design Solutions, Helsinki, Finland, 10-12 June.

Amor, R. and Faraj, I. 2001. Misconceptions about Integrated Project Databases, ITcon journal, ISSN 1400-6529, 6, http://www.itcon.org/2001/5/, 57-68.

Amor, R., Jiang, Y. and Chen, X. 2007. BIM in 2007 - are we there yet?, Proceedings of CIB W78 conference on Bringing ITC knowledge to work, Maribor, Slovenia, 26-29 June, 159-162.

Amor, R. and Ma, H. 2006. Preservation of Meaning in Mapped IFCs, Proceedings of EC-PPM 2006, Valencia, Spain, 13-15 September, 233-236.

Bentley 2009. Generative Components, web site last accessed 30/6/2009, US/Products/GenerativeComponents/.

Christiansson, P., Svidt, K. and Sorensen, K.B. 2008. Future Integrated Design Environments, Proceedings of CIB W78 conference on Improving the management of construction projects through IT adoption, Santiago, Chile, 15-17 July, 176-188.

CIB 2009. Integrated Design and Delivery Solutions, White Paper, CIB, The Netherlands.

Dimyadi, J., Amor, R. and Spearpoint, M. 2008. Sharing Building Information using the IFC Data Model for FDS Fire Simulation, Proceedings of 9th IAFSS Symposium on Fire Safety Science, Karlsruhe, Germany, 21-26 September.

Eastman, C. 1999. Building Product Models: Computer Environments, Supporting Design and Construction, CRC Press, ISBN-13: 978-0849302596, 424pp.

Eastman, C., Teicholz, P., Sacks, R. and Liston, K. 2008. BIM Handbook: A Guide to Building Information Modeling for Owners, Managers, Designers, Engineers and Contractors, Wiley, ISBN-13: 978-0470185285, 504pp.

Gray, J. and Reuter, A. 1993. Transaction processing: Concepts and Techniques, Morgan Kaufmann, ISBN-13: 978-155860-190-1, 1070pp.

Grilo, A., Jardim-Goncalves, R. and Steiger-Garcao, A. 2007. A methodology using domain ontology and SOA for better interoperability in AEC mass customization, Proceedings of CIB W78 conference on Bringing ITC knowledge to work, Maribor, Slovenia, 26-29 June.

Howard, R. 1998. Computing in Construction Pioneers and the Future, Butterworth Heinemann, ISBN 0-7507-3606-8, 138pp.

IAI 2009. International Alliance for Interoperability, web site last accessed 30/6/2009, http://www.iai-international.org/.

Kazi, A.S. and Wolf, P. 2006. Real-Life Knowledge Management Lessons from the Field, Knowledge Board, ISBN: 952-5004-72-4, 333pp.

Lipman, R.R. 2006. Mapping Between the CIMsteel Integration Standards (CIS/2) and Industry Foundation Classes (IFC) Product Model for Structural Steel, Proceedings of the conference on Computing in Civil and Building Engineering, Montreal, Canada, 14-16 June.

Ma, H., Ha, K.M.E., Chung, C.K.J. and Amor, R. 2006. Testing Semantic Interoperability, Proceedings of the Joint In- ternational Conference on Computing and Decision Making in Civil and Building Engineering. Montreal, Canada, 14-16 June.

NIST 2002. The Economic Impacts of Inadequate Infrastructure for Software Testing, Planning Report 02-3, NIST, May.

NIST 2004. Cost Analysis of Inadequate Interoperability in the U.S. Capital Facilities Industry, NIST GCR 04-867.

Open Design Alliance 2009. web site last accessed 30/6/2009, http://www.opendwg.org/.

Pazlar T. and Turk Z. 2006. Analysis of the geometric data exchange using the IFC, Proceedings of EC-PPM 2006, Valencia, Spain, 13-15 September, 165-171.

Preece, J., Rogers, Y. and Sharp, H. 2002. Interaction design: beyond human-computer interaction, John Wiley \& Sons, ISBN 0-471-49278-7, 519pp.

Solibri 2009. Solibri Model Checker v5, web site last accessed 30/6/2009, http://www.solibri.com/.

Sommerville, I. 2006. Software Engineering, Addison Wesley, ISBN-13: 978-0321313799, 864pp.

Turk, Ž. and Cerovšek, T. 2000 CONNET Software Center: A Section of the Construction Technology Park, Fruchter, R., Pena-Mora, F and Rodis, K (eds.) Computing in Civil and Building Engineering, Proceedings of the eighth international conference, ISBN 0-7844-0513-1, 325-332

Yurchyshyna, A., Zucker, C.F., Thanh, N.L., Lima, C. and Zarli, A. 2007. Towards an ontology-based approach for conformance checking modeling in construction, Proceedings of CIB W78 conference on Bringing ITC knowledge to work, Maribor, Slovenia, 26-29 June. 